\title{
SISTEMA DE MEDIÇÃO DE PERFORMANCE PARA O CONTROLE DE PLANTAS INVASORAS EM PLANTAÇÕES FLORESTAIS
}

\author{
Philipe Ricardo Casemiro Soares ${ }^{1}$, Romano Timofeiczyk Junior ${ }^{2}$, \\ João Carlos Garzel Leodoro da Silva², Marcos Milan ${ }^{3}$ \\ ${ }^{1}$ Universidade do Estado de Santa Catarina, Departamento de Engenharia Florestal, Lages, Santa Catarina, Brasil - \\ philipe.soares@udesc.br \\ ${ }^{2}$ Universidade Federal do Paraná, Departamento de Economia Rural e Extensão, Curitiba, Paraná, Brasil - romano@ufpr.br; \\ garzel@ufpr.br \\ ${ }^{3}$ Escola Superior de Agricultura Luiz de Queiroz, Universidade de São Paulo, Departamento de Engenharia de Biossistemas, \\ Piracicaba, São Paulo, Brasil - macmilan@usp.br
}

Recebido para publicação: 04/04/2014 - Aceito para publicação: 03/07/2014

\begin{abstract}
Resumo
Este trabalho objetivou a determinação de indicadores de desempenho (IDs) para a atividade de controle de plantas invasoras em plantações florestais. Para isso, tomaram-se como base as exigências de uma empresa atuante no segmento de celulose e papel, convertendo-as em um sistema de medição de performance, utilizando adaptações nas metodologias Desdobramento da Função Qualidade (QFD) e Balanced Scorecard (BSC). Foram definidos 19 indicadores de desempenho em cinco diferentes perspectivas: financeira; clientes; processos internos; aprendizado e crescimento; e não mercado. Destes, os indicadores que obtiveram maior peso foram dosagem de herbicida, condições dos equipamentos de aplicação, treinamento dos colaboradores e custo da qualidade relacionado à prevenção. Além disso, no geral os IDs apresentaram correlação positiva, em diferentes intensidades, indicando que melhorias em um grupo de indicadores podem influenciar positivamente os demais.

Palavras-chave: Indicadores de desempenho; balanced scorecard; desdobramento da função qualidade.
\end{abstract}

\begin{abstract}
Performance measurement system for weed control in forestry. This research aimed to determine performance indicators (PIs) for the activity of weed control in forest plantations. In order to that, the requirements of a company that act in the pulp and paper segment were converted into a performance measurement system, using adaptations of Quality Function Deployment (QFD) and Balanced Scorecard (BSC). We defined nineteen performance indicators in five different perspectives: financial; customer; internal process; learning and growth; and non-market. The indicators with the greatest weight were dose of herbicide, conditions of application equipment, training and quality costs related to prevention. Furthermore, in general, the PIs were positively correlated in different intensities, indicating that improvements in a group of indicators can influence others.

Keywords: Performance indicators; balance scorecard; quality function deployment.
\end{abstract}

\section{INTRODUÇÃO}

Em 2012, o Serviço Florestal Brasileiro (SFB) (2013) estimou que a área florestal do país era de 463 milhões de hectares, sendo aproximadamente $98,5 \%$ de florestas nativas e 1,5\% de plantadas. Considerando apenas os plantios florestais, o Brasil totalizou, nesse mesmo ano, uma área de aproximadamente 7,2 milhões de hectares. Destes, aproximadamente 93\% são plantios de espécies dos gêneros Eucalyptus e Pinus, acréscimo de 2,2\% em relação ao ano de 2011 (ASSOCIAÇÃO BRASILEIRA DE PRODUTORES DE FLORESTAS PLANTADAS (ABRAF), 2013).

Apesar da crescente área, especialmente do gênero Eucalyptus, a preocupação de empresas do setor florestal com a qualidade ainda pode ser considerada recente, com os primeiros trabalhos sendo realizados na década de 1980 (TRINDADE et al., 2012). Além disso, organizações que buscam a implementação de ferramentas da qualidade, comuns na área industrial, estão enfrentando dificuldades para adaptá-las ao seu processo silvicultural (TRINDADE et al., 2007). 
Esses problemas estão especialmente relacionados ao reduzido número de pesquisas que comprovem a viabilidade de aplicação e formas de adaptação de tais ferramentas à realidade enfrentada pelas organizações do setor florestal. Nesse sentido, são exemplos de estudos nas atividades de silvicultura e colheita documentados os trabalhos de Jacovine et al. (1999), Rezende et al. (2000), Milan et al. (2003), Leite et al. (2005) e Jacovine et al. (2005).

No ambiente mais competitivo em que as empresas florestais estão inseridas, a preocupação com a manutenção da qualidade do processo silvicultural é fundamental. Assim, para seu acompanhamento, é necessária uma base de informações bem estruturada, para auxiliar na tomada de decisão. Para isso, tornase importante o desenvolvimento de sistemas para a medição de desempenho que, de acordo com Macedo-Soares e Ratton (1999), devem ser alinhados aos objetivos estratégicos da organização.

Essas medições são realizadas por meio de indicadores de desempenho (IDs). No entanto, considerando toda a complexidade de uma organização, Epstein e Manzoni (1998) afirmam que indicadores isoladamente não permitem a mensuração total de sua performance. Dessa maneira, Kaplan e Norton (1992) propuseram o Balanced Scorecard (BSC), um sistema de medição de desempenho que não se baseia apenas em indicadores financeiros, mas também em outras três perspectivas: clientes; aprendizado e crescimento; e processos internos (KAPLAN; NORTON, 1996).

No entanto, em função do dinamismo do ambiente empresarial, um desses aspectos a serem incorporados ao Balanced Scorecard sugerido por Kaplan e Norton é a sustentabilidade (GATES; GERMAIN, 2010). São exemplos de adaptações do BSC as metodologias propostas por Figge et al. (2002) e Sidiropoulos et al. (2004), sendo a primeira denominada Sustainability Balanced Scorecard (SBSC), ou BSC Sustentável, e a segunda Eco-Balanced Scorecard.

Uma alternativa potencial para a definição dos indicadores de desempenho é o uso da metodologia Desdobramento da Função Qualidade (QFD), método empregado no desenvolvimento de produtos e serviços, garantindo a qualidade desde as fases iniciais dos projetos, pela tradução das exigências dos clientes em características técnicas mensuráveis (CARNEVALLI et al., 2004).

No setor florestal, essa metodologia foi utilizada por Milan et al. (2003) e por Matos (2009), respectivamente, para a obtenção de requisitos técnicos utilizados nas operações de preparo de solo mecanizado e de produção de mudas de espécies florestais.

Considerando a importância do tema, este trabalho tem como hipótese que, para o rigoroso controle da qualidade das operações florestais, é necessário um sistema de medição de performance bem estruturado. Assim, esta pesquisa teve como objetivo a definição de indicadores de desempenho para a atividade de controle de plantas invasoras de empresa atuante no setor florestal.

\section{MATERIAL E MÉTODOS}

Este trabalho foi desenvolvido em uma empresa florestal situada na região dos Campos Gerais, estado do Paraná, Brasil. A organização atua no segmento de celulose e papel e possui uma área de 135,2 mil hectares de efetivo plantio de espécies dos gêneros Pinus e Eucalyptus na região.

As atividades desenvolvidas na área florestal, exceto a colheita, até o final da pesquisa de campo eram terceirizadas, com o controle da qualidade da operação sendo realizado pelos próprios terceiros e, eventualmente, por auditorias de colaboradores da empresa. Visando o controle dessas atividades, uma das demandas da organização foi a identificação de indicadores de desempenho, necessidade que veio ao encontro dos objetivos deste estudo.

Dessa forma, o desenvolvimento da pesquisa foi realizado em duas etapas sequenciais: identificação dos fatores críticos de sucesso (FCS) para a atividade de controle de plantas invasoras e determinação e avaliação dos indicadores de desempenho.

\section{Identificação dos fatores críticos de sucesso}

Nessa fase, uma equipe de trabalho foi composta por colaboradores da empresa e pesquisador, posteriormente contando com o auxílio de membros externos. A equipe se reunia periodicamente, a princípio com a finalidade de definição dos FCS das operações florestais executadas pela empresa. Para isso, utilizaram-se como base as exigências do cliente (própria organização) para cada atividade, compondo, na matriz QFD, a qualidade desejada para os serviços prestados pelas terceiras, conforme descrito por Govers (1996), Adiano e Roth (1994) e Milan et al. (2003). 
Nesses encontros, utilizou-se a técnica de brainstorming, ou "tempestade de ideias", descrita por Trindade et al. (2007), em que todos os membros da equipe apresentavam suas ideias e o grupo chegava a suas conclusões. Esses fatores foram organizados em diagramas de afinidades (Figura 1), tendo seus respectivos graus de importância definidos pela equipe, em termos médios, com escala variando de 1 (pouco importante) a 5 (muito importante), para definição das qualidades desejada e planejada, seguindo metodologia proposta por Govers (1996). Em função da dificuldade de comparações, não foi considerado na determinação da qualidade planejada o benchmark.

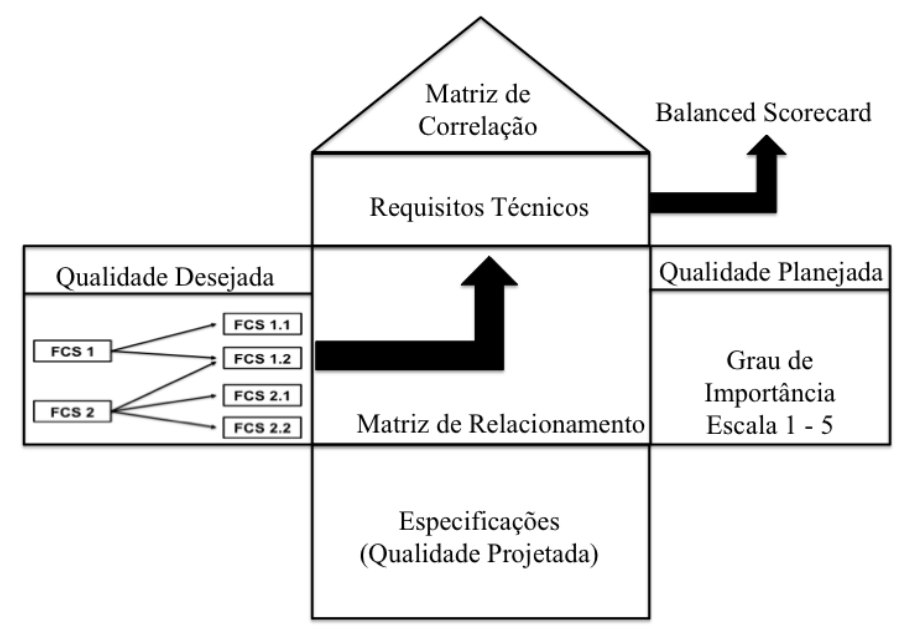

Figura 1. Esquema da matriz QFD.

Figure 1. Scheme of the QFD matrix.

Fonte: Adaptado de Govers (1996).

\section{Determinação dos indicadores de desempenho}

$\mathrm{Na}$ sequência, os fatores críticos de sucesso foram convertidos em requisitos técnicos (Figura 1) para a atividade de controle de plantas invasoras, conforme apresentado por Govers (1996). Para isso, mais uma vez foi utilizada a técnica de brainstorming com a equipe responsável, bem como a pesquisa em bibliografia e consulta de outros profissionais com conhecimento sobre a operação. Com isso, obtiveram-se os indicadores de desempenho para a mesma.

Nessa fase, utilizou-se também uma adaptação da metodologia Balanced Scorecard (BSC) desenvolvida por Kaplan e Norton (1992). Assim, os indicadores de desempenho definidos para as diferentes atividades florestais foram agrupados em cinco perspectivas, as quatro originais descritas pelos autores (financeira, clientes, processos internos, aprendizado e crescimento) e uma adicional, denominada neste estudo de "não mercado", sendo alocados os requisitos que não estavam relacionados a nenhuma das demais perspectivas, como descrito por Figge et al. (2002). Um esquema da definição dos indicadores de desempenho é apresentado na figura 2.

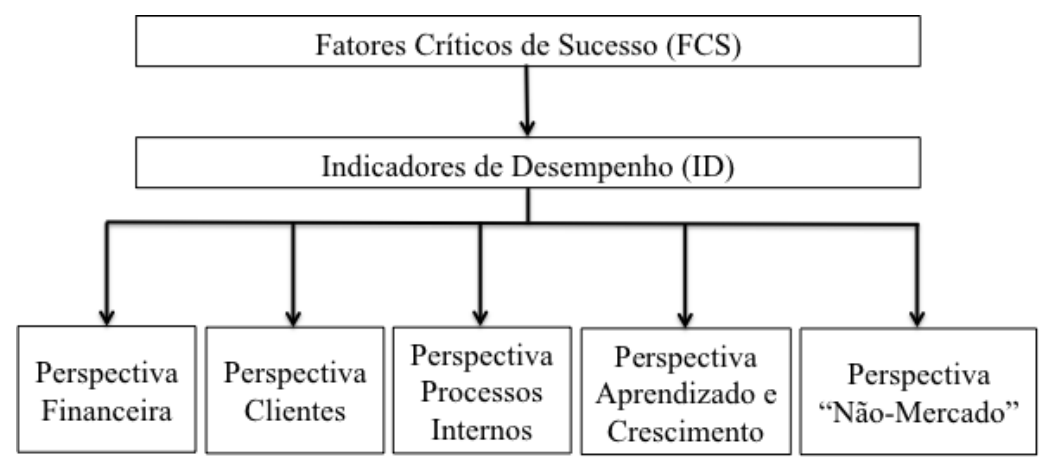

Figura 2. Esquema da definição dos indicadores de desempenho com a metodologia BSC.

Figure 2. Scheme of the definition of the performance indicator with BSC methodology.

FLORESTA, Curitiba, PR, v. 45, n. 1, p. 175 - 184, jan. / mar. 2015.

Soares, P. R. C. et al.

ISSN eletrônico 1982-4688 / ISSN impresso 0015-3826 
Para essa etapa, buscou-se manter uma relação entre os indicadores alocados nas diferentes perspectivas. Dessa maneira, melhorias em IDs de determinada perspectiva devem impactar positivamente nas medidas de performance das demais.

Em seguida, as relações existentes entre os indicadores de desempenho foram avaliadas a partir da matriz de correlação que representa o "telhado" da casa da qualidade, resultado da aplicação e organização das informações analisadas pela metodologia QFD. Para isso, adaptou-se a metodologia descrita por Govers (1996), em que a análise é realizada por meio de símbolos, avaliando-se originalmente as relações em positiva ou relativa de diferentes graus.

No entanto, para este trabalho, verificou-se somente a existência de influência positiva entre os indicadores, ou seja, se melhorias em um requisito técnico afetariam positivamente os demais, além da intensidade da relação. Nesse caso, considerou-se como correlação forte aquela existente entre indicadores que se influenciam de maneira direta. Foi dita moderada quando a influência entre os IDs ocorria de maneira indireta. Por fim, foi considerada como correlação fraca as medidas de desempenho que pouco se influenciam. No caso de não existência de correlação entre os indicadores, a matriz não era preenchida.

Na sequência, analisou-se a correlação entre os fatores críticos de sucesso e os indicadores de desempenho na matriz de relacionamento, de acordo com a metodologia "9-3-1" apresentada por Govers (1996). Em caso de não existir nenhuma relação, o espaço na matriz era deixado em branco.

Essa metodologia permite atribuir um peso para cada relação FCS-ID, com a finalidade de destacar os principais indicadores para os processos silviculturais da empresa avaliada, denominada qualidade projetada, determinada a partir do produto entre o grau de importância, anteriormente definido, e o peso da relação entre fator crítico de sucesso e medida de desempenho, como na equação 1 (MILAN et al., 2003).

$$
\mathrm{W}_{\mathrm{ij}}=\mathrm{GI}_{\mathrm{i}} \quad \mathrm{P}_{\mathrm{ij}}
$$

em que: $\mathrm{W}_{\mathrm{ij}}=$ peso absoluto do relacionamento entre o i-ésimo FCS e o j-ésimo ID;

$\mathrm{GI}_{\mathrm{i}}=$ grau de importância do i-ésimo FCS; e

$\mathrm{P}_{\mathrm{ij}}=$ peso da relação entre o i-ésimo FCS e o j-ésimo ID, de acordo com metodologia 9-3-1.

Finalizando o processo de identificação dos indicadores de desempenho, foram determinados os pesos absolutos e relativos de cada um deles. O peso absoluto da medida é obtido pelo somatório de seus respectivos pesos absolutos de relacionamento com os fatores críticos de sucesso (Wij), como demonstrado Milan et al. (2003).

$$
\mathrm{AW}_{\mathrm{j}}={ }_{\mathrm{i}=1}^{\mathrm{n}} \mathrm{W}_{\mathrm{ij}}
$$

em que: $\mathrm{AWj}=$ peso absoluto do j-ésimo ID.

Já o peso relativo, em porcentagem, de cada medida de desempenho foi estimado pela equação 3 .

$$
\mathrm{RW}_{\mathrm{j}}=\frac{\mathrm{AW}_{\mathrm{j}}}{\mathrm{n} \mathrm{AW}_{\mathrm{j}}} 100
$$

em que: $\mathrm{RWj}$ = peso relativo do j-ésimo ID.

Esses pesos permitiram identificar quais os indicadores mais importantes e críticos para o sucesso das diferentes operações silviculturais desenvolvidas pela empresa florestal e, dessa maneira, a qualidade projetada para o sistema a ser desenvolvido. Na sequência, realizou-se a avaliação dos possíveis desvios nos indicadores definidos nessa fase do trabalho. 


\section{RESULTADOS E DISCUSSÃO}

\section{Identificação dos fatores críticos de sucesso (FCS)}

Para essa etapa, foram considerados como fatores críticos de sucesso aspectos definidos como importantes pela empresa para atingir as metas de cada atividade. Em função dos resultados obtidos nesta pesquisa, os fatores considerados como primários para todas as atividades foram eficiência, economia e aspectos ambientais.

Assim, o diagrama de afinidades para a atividade de controle de invasoras é apresentado na figura 3. Relaciona-se com a eficiência "controlar mato-competição", objetivo da operação para melhorar o desenvolvimento da floresta, fato evidenciado pelos trabalhos de Aparício et al. (2010) e Londero et al. (2012), por isso com maior grau de importância $(\mathrm{GI}=5,0)$. Além disso, relaciona-se com "evitar danos às mudas" (GI $=4,3)$ e "seguir os procedimentos operacionais" $(\mathrm{GI}=3,6)$, que auxiliam no alcance desse objetivo.

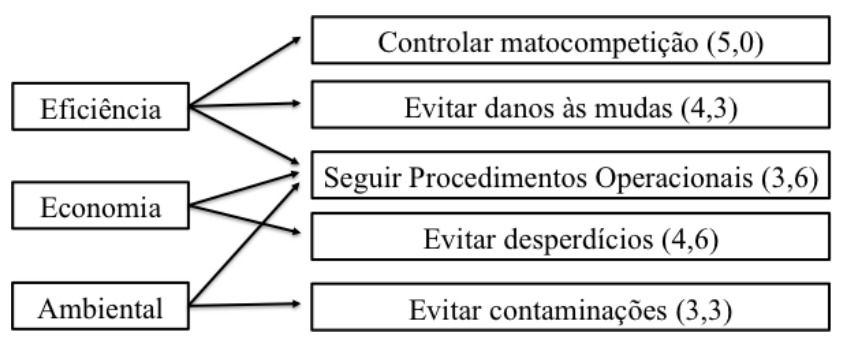

Figura 3. Diagrama de afinidades e graus de importância para os FCS da operação de controle de plantas invasoras.

Figure 3. Diagram of affinity and importance rating for the FCS of weed control.

Os fatores críticos de sucesso de segundo nível que se relacionam à economia foram "seguir os procedimentos operacionais" e "evitar desperdícios", este com maior grau de importância $(4,6)$, por permitir a redução de custos de maneira direta. Ambos permitem a melhoria nos indicadores operacionais (STAPE, 1997; LIKER, 2005). Já para as questões ambientais, destacam-se "evitar contaminações" $(\mathrm{GI}=3,3)$ e "seguir procedimentos operacionais", com o objetivo de reduzir os impactos negativos das atividades. Nesse caso, os problemas ambientais estão principalmente relacionados ao vazamento de combustível e lubrificante das máquinas utilizadas na operação, uma vez que as empresas florestais utilizam herbicida de baixa toxicidade, em função de aspectos relacionados à certificação.

Os procedimentos operacionais são fundamentais para as atividades florestais no geral. $\mathrm{O}$ fato de a empresa detalhar suas operações, gerenciando e padronizando sua rotina, permite a aproximação da eficiência, seja ela técnica, econômica ou ambiental (CAMPOS, 2004). Dessa maneira, o cumprimento desses procedimentos pelos colaboradores facilita o sucesso da organização, estando relacionado a todos os demais FCS, independentemente do nível.

\section{Determinação dos indicadores de desempenho}

No total, 19 indicadores para a operação de controle de plantas invasoras foram definidos nas perspectivas do BSC (Tabela 1). A matriz QFD utilizada para definição dos indicadores da atividade, bem como sua correlação com os fatores críticos de sucesso e respectivos graus de importância (qualidade exigida e planejada), além de seus pesos absolutos e relativos (qualidade projetada) para a definição das medidas de desempenho, é apresentada na figura 4.

$\mathrm{Na}$ perspectiva financeira, destaca-se o custo operacional $\left(\mathrm{R} \$ \cdot \mathrm{ha}^{-1}\right)$ ou o valor total gasto com a operação. Nesse caso, devem ser computadas as saídas de caixa referentes a insumos, mão de obra e maquinário utilizado na atividade, além de outros gastos, como aqueles apresentados por Graça et al. (2000). Esse valor deve ser comparado à meta da empresa e, considerando um desvio aceitável, pode gerar uma não conformidade. Esse indicador, na análise QFD, obteve um peso relativo de 4,6\%, mostrando sua importância pela correlação com todos os fatores críticos de sucesso.

Além disso, por ser avaliada a qualidade das atividades, também foi considerado como indicador o custo da qualidade. Para isso, utilizou-se como base a divisão apresentada por Jacovine et al. (1999) e Leite et al. (2005), em que tais custos são divididos em prevenção, avaliação ou monitoramento e falhas (interna e externa). 


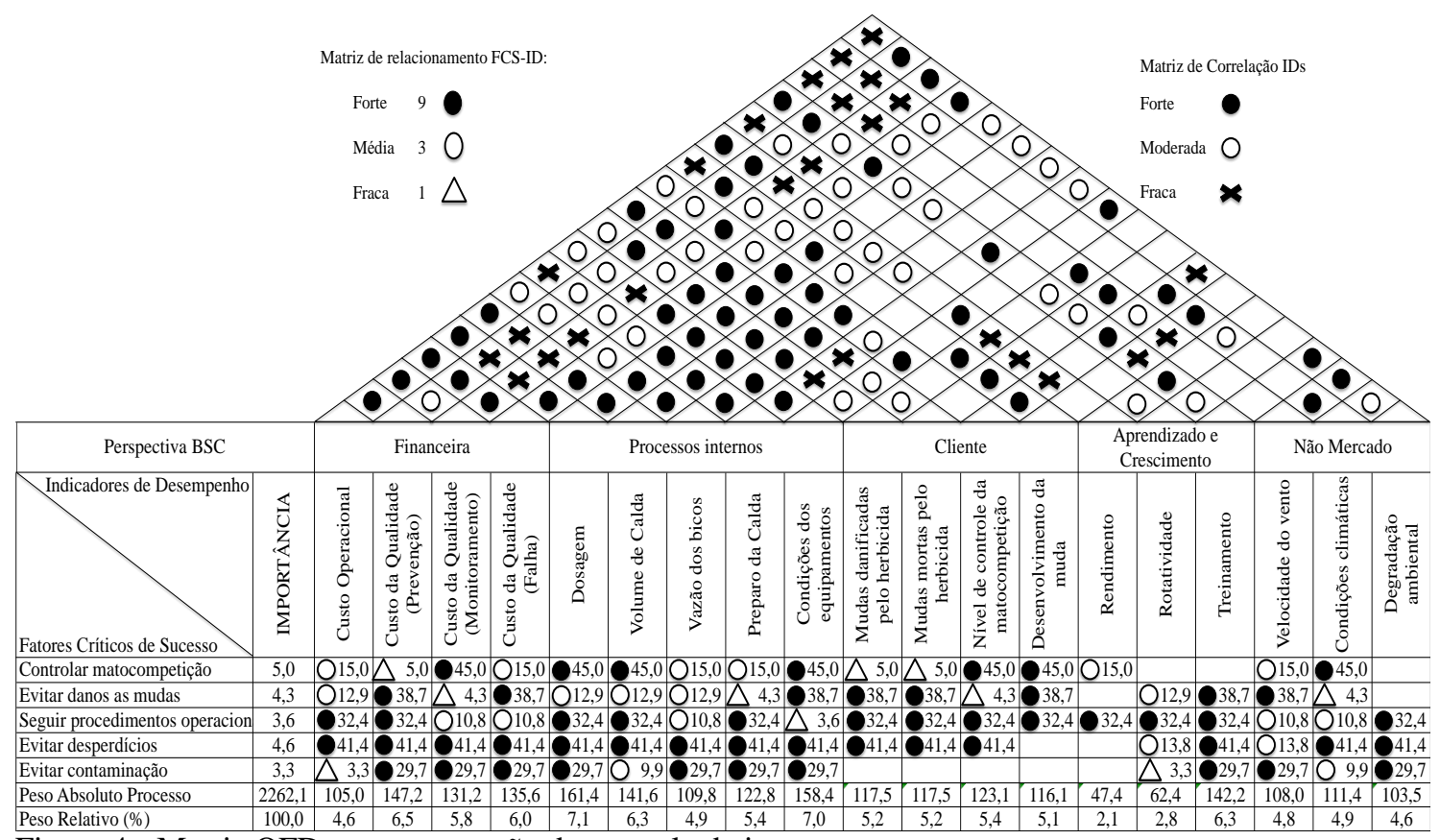

Figura 4. Matriz QFD para a operação de controle de invasoras.

Figure 4. QFD matrix for the activity of weed control.

Tabela 1. Indicadores de desempenho da operação de controle de invasoras nas perspectivas da metodologia BSC adaptada.

Table 1. Performance indicators of weed control in adapted BSC perspectives.

\begin{tabular}{|c|c|c|}
\hline \multirow{2}{*}{ Perspectiva } & \multicolumn{2}{|c|}{$\begin{array}{r}\text { Controle de plantas invasoras } \\
\end{array}$} \\
\hline & Indicador de desempenho & Medição \\
\hline \multirow{4}{*}{ Financeira } & Custo operacional & $\mathrm{R} \$$ \\
\hline & Custo da qualidade (prevenção) & $\mathrm{R} \$$ \\
\hline & Custo da qualidade (monitoramento) & $\mathrm{R} \$$ \\
\hline & Custo da qualidade (falhas) & $\mathrm{R} \$$ \\
\hline \multirow{5}{*}{ Processos internos } & Dosagem & Kg.ha $^{-1}$ \\
\hline & Volume de calda & L.ha ${ }^{-1}$ \\
\hline & Vazão dos bicos & $\mathrm{L}$ \\
\hline & Preparo de calda & Conforme/Não conforme \\
\hline & Condições dos equipamentos & Conforme/Não conforme \\
\hline \multirow{4}{*}{ Clientes } & Mudas danificadas & $\%$ \\
\hline & Mudas mortas & $\%$ \\
\hline & Nível de mato-competição & Escala 1-4 \\
\hline & Desenvolvimento da muda & Conforme/Não conforme \\
\hline \multirow{3}{*}{$\begin{array}{l}\text { Aprendizado e } \\
\text { crescimento }\end{array}$} & Rendimento & HH.ha $^{-1}$ ou HM.ha ${ }^{-1}$ \\
\hline & Treinamento & Horas \\
\hline & Rotatividade & Número tempo ${ }^{-1}$ \\
\hline \multirow{3}{*}{ Não mercado } & Velocidade do vento & Conforme/Não conforme \\
\hline & Condições climáticas & Conforme/Não conforme \\
\hline & Degradação ambiental & Conforme/Não conforme \\
\hline
\end{tabular}

O custo de prevenção é o gasto da empresa para evitar não conformidades no processo, enquanto o de monitoramento é aquele relacionado à verificação do grau de conformidade do produto ou processo. Já os custos de falhas são devidos aos desperdícios gerados por erros no processo produtivo (GRYNA, 1998). 
Esses indicadores também podem ser considerados importantes na atividade analisada, obtendo pesos relativos de 6,5\% para prevenção, 5,8\% para monitoramento e 6,0\% para custos de falhas. Destacase a maior importância para os gastos de prevenção, uma vez que eles permitem a redução dos demais relacionados à qualidade.

Em uma empresa deve haver um equilíbrio dos gastos com a qualidade em relação ao previsto, para que não haja grandes desvios no custo operacional em função da alta correlação entre eles. Além disso, para Jacovine et al. (1999), considerando-se apenas os custos relacionados às falhas, a organização deve sempre buscar uma forma de minimizá-lo, sendo uma alternativa o investimento na qualidade.

Já os indicadores definidos, considerando a perspectiva aprendizado e crescimento, foram o rendimento da atividade, a rotatividade de colaboradores ou turnover e seu treinamento. $\mathrm{O}$ rendimento (peso relativo de $2,1 \%$ ), medida em hora-homem ou hora-máquina por hectare, deve ser comparado a um padrão definido pela empresa para a operação. Além disso, é importante o constante acompanhamento desse indicador, verificando-se a possibilidade e a efetividade de melhorias que possam gerar economia para a empresa.

Também relacionada à perspectiva, a rotatividade (peso relativo de $2,8 \%$ ) indica a taxa de substituição de trabalhadores em intervalos de tempo a serem definidos pela empresa. Esse indicador pode ser relacionado ao comportamento ou atitude do colaborador, bem como a fatores motivacionais. Independentemente da causa, o turnover gera um aumento de custos para as empresas, relacionado ao desligamento, recrutamento, seleção e treinamento (CHIAVENATO, 2008). Além disso, outro fato negativo gerado pelo turnover é a perda do conhecimento acumulado pelo colaborador durante o tempo em que esteve na empresa.

Ambos os indicadores obtiveram pesos menores. No entanto, a importância total da perspectiva foi balanceada com as demais em função do treinamento, medido em horas, que pode ser considerado fundamental para o sucesso do processo, com peso relativo de 6,3\%. Esse indicador está correlacionado com grande parte dos fatores críticos de sucesso e demais medidas de desempenho. $\mathrm{O}$ fato de a empresa treinar seus colaboradores permite melhorias nos demais ID, como por exemplo o aumento do rendimento e a redução dos custos de qualidade, especialmente aqueles relacionados às falhas no processo.

Definiram-se, para a perspectiva processos internos, os indicadores dosagem, volume de calda, vazão dos bicos, preparo de calda e condições dos equipamentos. A dosagem foi a medida de desempenho que apresentou maior peso relativo para a perspectiva $(7,1 \%)$, por ser fundamental para o controle da mato-competição, fator crítico de sucesso com maior grau de importância para a atividade. Além disso, a dosagem apresenta correlação forte a moderada com os demais FCS da operação e com grande parte de seus indicadores de desempenho.

Outro indicador considerado importante para o controle de plantas invasoras foi o volume de calda, com peso relativo de $6,3 \%$, seguindo padrão de correlação semelhante àquele apresentado pela dosagem, tanto para fatores críticos de sucesso, especialmente o controle da mato-competição, quanto para as demais medidas de desempenho definidas para a operação.

Ambos os indicadores, dosagem e volume de calda, devem ter um padrão e desvio tolerável, definidos pela empresa, para comparação e verificação de não conformidades. A dosagem realizada é determinada com o acompanhamento do preparo e do volume de calda. Já o segundo indicador é calculado a partir da vazão dos bicos, outra medida de desempenho definida para a operação alocada na perspectiva processos internos. A vazão dos bicos é utilizada para cálculo do volume de calda realizado e, portanto, para a efetividade no controle da mato-competição (peso relativo de 4,9\%).

Fechando os indicadores na perspectiva processos internos estão a verificação da conformidade no preparo de calda e das condições dos equipamentos, com pesos relativos de 5,4\% e 7,0\%, respectivamente. A primeira medida de desempenho consiste na observação do cumprimento dos procedimentos operacionais, e a segunda na verificação dos equipamentos para a aplicação de herbicidas, como entupimentos, entre outros fatores.

Já para a perspectiva dos clientes foram definidos como IDs as porcentagens de mudas mortas ou danificadas pelo herbicida, o nível de mato-competição e o desenvolvimento das mudas. Esta última medida de desempenho apresentou peso relativo de $5,1 \%$, sendo definida pela verificação da conformidade no crescimento das mudas, considerando-se um padrão desejado e realista, determinado em função da espécie e procedência, além de condições ambientais e de manejo florestal.

FLORESTA, Curitiba, PR, v. 45, n. 1, p. 175 - 184, jan. / mar. 2015. 
Outro indicador qualitativo para essa perspectiva (peso relativo de 5,4\%) foi o nível da matocompetição, avaliação direta da eficácia dessa atividade e fundamental para a determinação da necessidade de novas aplicações de herbicida na área. Para essa verificação, é sugerida a divisão em quatro níveis (Tabela 2), em função da porcentagem da área com espécies vegetais que possam prejudicar o desenvolvimento da plantação, com a não conformidade determinada pela tolerância estipulada pela empresa.

Tabela 2. Escala para avaliação do nível de mato-competição.

Table 2. Scale for evaluation of the level of weed competition.

\begin{tabular}{ll}
\hline Valor & Descrição \\
\hline 1 & Presença de espécies invasoras em até $25 \%$ do talhão \\
2 & Presença de espécies invasoras em até 50\% do talhão \\
3 & Presença de espécies invasoras em até 75\% do talhão \\
4 & Presença de espécies invasoras em 100\% do talhão \\
\hline
\end{tabular}

Formam o grupo de medidas de desempenho quantitativas alocadas nessa perspectiva a porcentagem de mudas danificadas ou mortas pela ação do herbicida, com peso relativo de $5,2 \%$ cada. Nesses casos, o uso de herbicidas seletivos reduz o risco de perdas de produtividade em função de não conformidades nessas medidas de desempenho.

Alocaram-se também indicadores em perspectiva adicional, denominada de "não mercado", sendo eles e seus respectivos pesos relativos: velocidade do vento $(4,8 \%)$, condições climáticas $(4,9 \%)$ e degradação ambiental (4,6\%). A avaliação deles é realizada pela observação de não conformidades durante a execução da atividade.

Consideraram-se os aspectos climáticos pela interferência nos fatores críticos de sucesso. Nesse sentido, a velocidade do vento pode levar a deriva do herbicida (SHIRATSUCHI; FONTES, 2002), aumentando o risco de danos às mudas e de contaminação ambiental. Esses problemas podem ser amenizados com o uso de produtos seletivos e de baixa toxicidade. Além disso, fatores como temperatura, umidade e precipitação podem reduzir a eficiência da operação, gerando desperdícios (PENCKOWSKI et al., 2003).

No geral, os indicadores são correlacionados de maneira média a forte, comportamento esperado em função da utilização da metodologia BSC. Assim, melhorias em um dos IDs definidos podem impactar positivamente outras medidas de desempenho, mesmo aquelas alocadas em outras perspectivas do Balanced Scorecard.

\section{CONCLUSÕES}

- Foram identificados 19 indicadores de desempenho para a atividade de controle de plantas invasoras em povoamentos florestais, alocados em cinco diferentes perspectivas do Balanced Scorecard. No geral, tais medidas de desempenho apresentaram correlação com as demais, indicando que melhorias em uma teriam impactos positivos sobre as outras.

- Quanto ao desenvolvimento das medidas de performance, a divisão nas cinco perspectivas, além de permitir o acompanhamento do desempenho passado da empresa, possibilita o entendimento de seu presente e a projeção do futuro. Dessa maneira, o uso do BSC se mostrou fundamental para aumentar a eficiência no controle da operação.

- Assim, sugere-se à empresa a adoção de tal sistema de medição de performance para o acompanhamento da qualidade da atividade de controle de plantas invasoras em plantações florestais. Além disso, é importante a ampliação do sistema, visando o controle das demais atividades do processo silvicultural da organização, bem como a definição dos limites de tolerância para alguns dos indicadores, especialmente os quantitativos, em função de sua capacidade de processo.

- No entanto, o uso dos indicadores definidos para a atividade de controle de plantas invasoras em plantios florestais por outras empresas do setor, apesar de aconselhável, exige pesquisas visando a adaptação, em função de diferentes exigências que possam existir, de acordo com a realidade enfrentada pela organização. 


\section{REFERÊNCIAS}

ADIANO, C.; ROTH, A. V. Beyond the house of quality: dynamic QFD. Benchmarking for Quality Management and Technology, [s.l.], v. 1, n. 1, p. 25 - 37, 1994.

APARÍCIO, P. S.; FERREIRA, R. L. C.; SILVA, J. A. A. da; ROSA, A. C.; APARÍCIO, W. C. S. Controle da mato-competição em plantios de dois clones de Eucalyptus urograndis no Amapá. Ciência Florestal, Santa Maria, v. 20, n. 3, p. 381 - 390, 2010.

ASSOCIAÇÃO BRASILEIRA DE PRODUTORES DE FLORESTAS PLANTADAS (ABRAF). Anuário estatístico ABRAF 2013: Ano-base 2012. Brasília: ABRAF, 2013. 148 p.

CAMPOS, V. F. Gerenciamento da rotina do trabalho do dia a dia. Nova Lima: INDG, 2004. 266 p.

CARNEVAlLI, J. A.; SASSI, A. C.; MIGUEL, P. A. C. Aplicação do QFD no desenvolvimento de produtos: levantamento sobre seu uso e perspectivas para pesquisas futuras. Gestão \& Produção, São Carlos, v. 11, n. 1, p. 33 - 49, 2004.

CHIAVENATO, I. Gestão de pessoas. 3. ed. rev. e atual. Rio de Janeiro: Elsevier, 2008. 579 p.

EPSTEIN, M.; MANZONI, J. F. Implementing corporate strategy: from tableaux de bord to balanced scorecards. European Management Journal, Oxford, v. 16, n. 2, p. 190 - 203, 1998.

FIGGE, F.; HAHN, T.; SCHALTEGGER, S.; WAGNER, M. The sustainability balanced scorecard: linking sustainability management to business strategy. Business Strategy and the Environment, New York, v. 11, n. 5, p. 269 - 284, 2002.

GATES, S.; GERMAIN, C. Integrating sustainability measures into Strategic Performance Measurement Systems: an empirical study. Management Accounting Quarterly, Montvale, v. 11, n. 3, p. 1-7, 2010.

GOVERS, C. P. M. What and how about quality function deployment (QFD). International Journal of Production Economics, Amsterdam, v. 46-47, p. 575 - 585, 1996.

GRAÇA, L. R.; RODIGHERI, H. R.; CONTO, A. J. de. Custos florestais de produção: conceituação e aplicação. Colombo: Embrapa Florestas, 2000. 32 p.

GRYNA, F. M. Quality and Costs. In: JURAN, J. M.; GODFREY, A. B. Juran's quality Handbook. New York: McGraw Hill, 1998. p. 8. 1 - 8.26.

JACOVINE, L. A. G.; REZENDE, J. L. P.; SOUZA, A. P. de; LEITE, H. G.; TRINDADE, C. Descrição e uso de uma metodologia para avaliação dos custos de qualidade na colheita florestal semimecanizada. Ciência Florestal, Santa Maria, v. 9, n. 1, p. 143 - 160, 1999.

JACOVINE, L. A. G.; MACHADO, C. C.; SOUZA, A. P. de; LEITE, H. G.; MINETTI, L. J. Avaliação da qualidade operacional em cinco subsistemas de colheita florestal. Revista Árvore, Viçosa, v. 29, n. 3, p. $391-400,2005$.

KAPLAN, R. S.; NORTON, D. P. The balanced scorecard - measures that drive performance. Harvard Business Review, Boston, p. 69 - 79, 1992.

The Balanced Scorecard: translating strategy into action. Boston: Harvard Business School Press, 1996. 322 p.

LEITE, H. G.; JACOVINE, L. A. G.; SILVA, C. A. B. da; PAULA, R. A. de; PIRES, I. E.; SILVA, M. L. da. Determinação dos custos da qualidade em produção de mudas de eucalipto. Revista Árvore, Viçosa, v. 29, n. 6, p. 955 - 964, 2005.

LIKER, J. K. O modelo Toyota: 14 princípios de gestão do maior fabricante do mundo. Porto Alegre: Bookman, 2005. 316 p. 
LONDERO, E. K.; SCHUMACHER, M. V.; RAMOS, L. O. O.; RAMIRO, G. A.; SZYMCZAK, D. A. Influência de diferentes períodos de controle e convivência de plantas daninhas em eucalipto. Cerne, Lavras, v. 18, n. 3, p. 441 - 447, 2012.

MACEDO-SOARES, T. D. L. V. A. de; RATTON, C. A. Medição de desempenho e estratégias orientadas para o cliente: resultados de uma pesquisa de empresas líderes no Brasil. Revista de Administração de Empresas, São Paulo, v. 39, n. 4, p. 46 - 59, 1999.

MATOS, R. B. de. Planejamento da qualidade para o processo de produção de mudas clonais de eucalipto. 74 f. Tese (Doutorado em Recursos Florestais) - Escola Superior de Agricultura "Luiz de Queiroz", Universidade de São Paulo, Piracicaba, 2009.

MILAN, M.; BARROS, J. W. D.; GAVA, J. L. Planning soil tillage using Quality Function Deployment (QFD). Scientia Agricola, Piracicaba, v. 60, n. 2, p. 217 - 221, 2003.

PENCKOWSKI, L. H.; PODOLAN, M. J.; LÓPEZ-OVEJERO, R. F. Influência das condições climáticas no momento da aplicação de herbicidas pós-emergentes sobre a eficácia do controle da nabiça (Raphanus raphanistrum) na cultura do trigo. Planta Daninha, Viçosa, v. 21, n. 3, p. 435 - 442, 2003.

REZENDE, J. L. P.; JACOVINE, L. A. G.; LEITE, H. G.; TRINDADE, C. Avaliação da qualidade na colheita florestal semimecanizada. Scientia Forestalis, Piracicaba, SP, n. 57, p. 13 - 26, jun. 2000.

SERVIÇO FLORESTAL BRASILEIRO (SFB). Florestas do Brasil em resumo - 2013: dados de 20072012. Brasília: SFB, 2013. 188 p.

SIDIROPOULOS, M.; MOUZAKITIS, Y.; ADAMIDES, E.; GOUTSOS, S. Applying sustainable indicators to corporate strategy: the Eco-Balanced Scorecard. Environmental Research, Engineering and Management, Kaunas, v. 27, n. 1, p. 28 - 33, 2004.

SHIRATSUCHI, L. S.; FONTES, J. R. A. Tecnologia de aplicação de herbicidas. Planaltina: Embrapa Cerrados, 2002. $30 \mathrm{p}$.

STAPE, J. L. Planejamento global e normatização de procedimentos operacionais da talhadia simples em Eucalyptus. Série Técnica IPEF, Piracicaba, v. 11, n. 30, p. 51 - 62, 1997.

TRINDADE, C.; JACOVINE, L. A. G.; REZENDE, J. L. P.; SARTÓRIO, M. L. Gestão e controle da qualidade na atividade florestal. Viçosa: Editora UFV, 2012. 253 p.

TRINDADE, C.; REZENDE, J. L. P.; JACOVINE, L. A. G.; SARTÓRIO, M. L. Ferramentas da qualidade: aplicação na atividade florestal. 2. ed. Viçosa: Editora UFV, 2007. 159 p. 